\begin{tabular}{|l|l|c|c|}
\hline Eiszeitalter u. Gegenwart & $\mathbf{2 9}$ & $\begin{array}{c}179-188 \\
6 \mathrm{Abb} .\end{array}$ & Hannover 1979 \\
\hline
\end{tabular}

\title{
Tunneltäler in Dänemark
}

\author{
STEEN SJÖRRING *)
}

Upper Pleistocene (Saale and Weichsel glaciation), "tunnel valley “, channel, fluviatile erosion, glacial erosion, geomorphologic sketch map Denmark

Kurzfassung: Der Begriff "Tunneltal“ umfaßt mehrere verschiedene morphologische Elemente. Neue Ergebnisse weisen darauf hin, daß die klassische Tunneltalhypothese aufgegeben werden muß. Einige der Tunneltäler sind wahrscheinlich als präglaziale oder proglaziale Wasserläufe angelegt und sind später durch selektive Glazialerosion überprägt worden.

\section{[Tunnel Valleys in Denmark]}

A bstract. The term "tunnel valley" comprises more different geomorphological elements. Based on new investigations it appears that the hypothesis for formations of tunnel valleys cannot longer be maintained. Some of the tunnel valleys may have an origin as preglacial or proglacial streems, which later on has been deepend by selective glacial erosion.

In einem größtenteils aus Lockersedimenten aufgebauten Flachland wie Dänemark ist die Ausformung der Oberfläche vor allem auf die Wirkung des Inlandeises und seiner Schmelzwässer zurückzuführen.

Auf Abb. 1 sind einige geomorphologische und quartärgeologische Grenzlinien eingezeichnet. Die westliche Linie muß im Wesentlichen als Grenze der Eisbedeckung der letzten Glazialzeit (Weichsel/Würm) betrachtet werden.

Es besteht ein sehr deutlicher geomorphologischer Unterschied zwischen den Gebieten beiderseits dieser Grenzlinie, die die Weichsel-Hauptstillstandslinie (oder Ussings Eisrandlinie) genannt wird. Auf der westlichen Seite liegen die großen westjütländischen Sander, die nur von den saale-(riß-)zeitlichen „b a k k eø e r ", die man in direkter Übersetzung aus dem Dänischen als „Hügelinseln“ bezeichnen könnte, unterbrochen werden. Auf der östlichen Seite der Grenzlinie sind die Glazialformen in ihrem Jugendistadium - mit Moränenflächen, Hügellandschaften und Tunneltälern — gut erhalten.

Die östlichste Grenzlinie, auch als die HARDER'sche Eisrandlage bekannt, kann als Ausbreitungsgrenze des letzten jungbaltischen Eisstromes betrachtet werden.

Auf der Abb. 1 sind auch die wichtigsten Tunneltäler in großen Zügen eingezeichnet. Die Tunneltäler sind von Ussing (1903, 1904 und 1907) als „f jordda le “ (direkt übersetzt: Fjord- oder Fördentäler) bezeichnet worden. In seinen Arbeiten über die Sandermorphologie Westjütlands entdeckte Ussing, daß die Sander flache Halbkegel bilden, deren höchster Punkt dort liegt, wo die Fördentäler enden. Deshalb schien eine genetische Verknüpfung der Sander mit den Fördentäler nahezuliegen. Ussıng ließ jedoch die Frage offen, ob die Fördentäler von subglazialen oder supraglazialen Schmelzwässern geschaffen worden waren. Später aber hat MADSEN (1921), übereinstimmend mit WoldstedT (1913), eine subglaziale Entstehung der Fördentäler festgestellt. Um alle Zweifel auszuräumen, hat MADSEN gleichzeitig den Namen „Tunneltal“ (dänisch: „tunneldal“) eingeführt.

*) Anschrift des Verfassers: Univ.lektor S. S j ör ring, Institut for almen Geologi, Köbenhavns Universitet O'stervoldgade 10, DK-1350 Köbenhavn K, Dänemark. 
Der Begriff „Tunneltal“ umfaßt mehrere verschiedene morphologische Elemente, die wahrscheinlich unterschiedlicher Entstehung sind. Die dänischen Tunneltäler haben Längen von 5 bis $70 \mathrm{~km}$. Ahnlich wie die Tunneltäler in Norddeutschland zeigen sie ein unregelmäßiges Bodenrelief. Stellenweise sind sie durch Schwellen unterbrochen und/oder

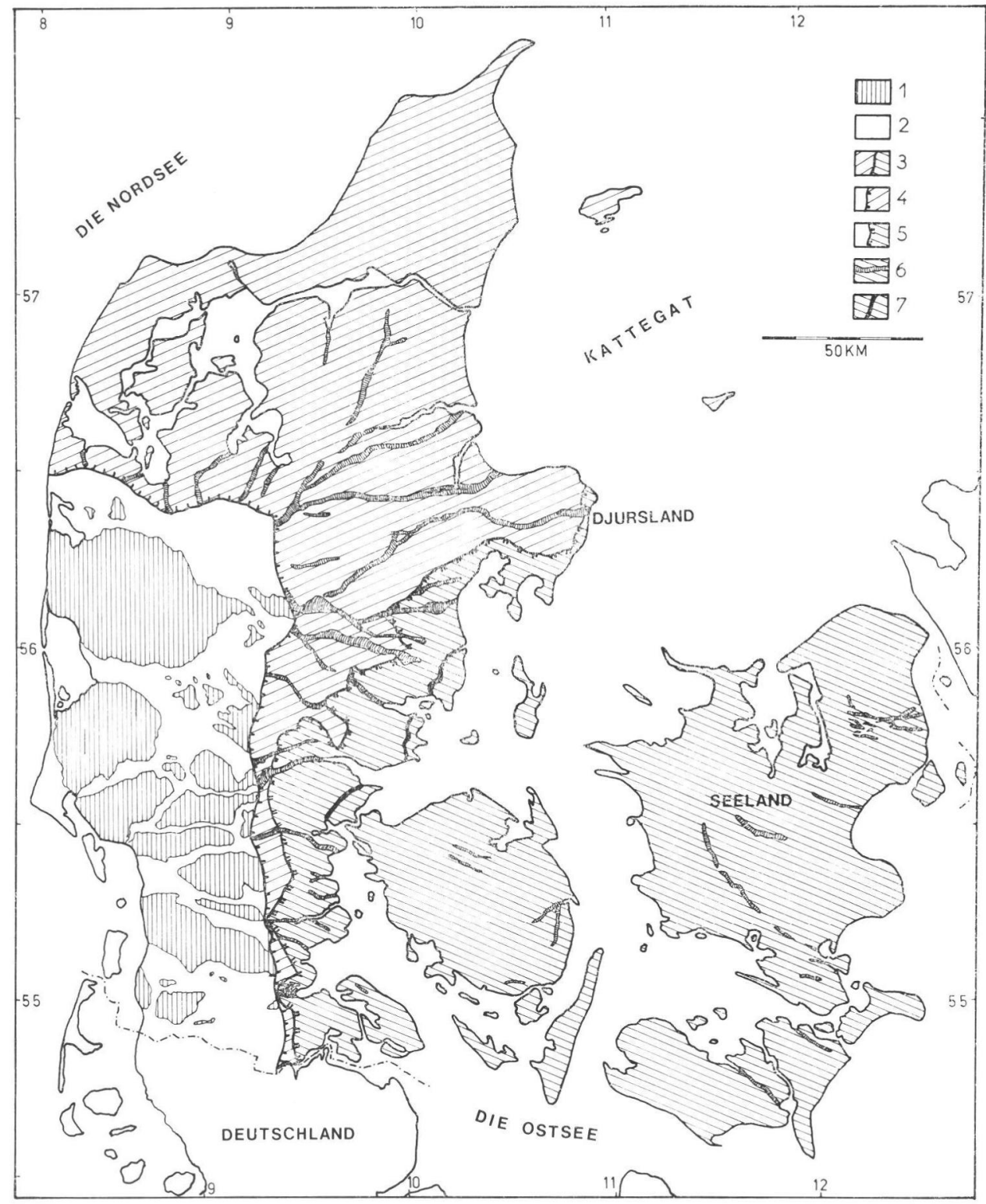

Abb. 1: Vereinfachte geomorphologische Karte (frei nach K. Mirthers 1942). Zeichenerklärung: 1. Saale-(Riß-)zeitliche Landschaft, 2. Sander der Weichsel-(Würm-)eiszeit, 3. Weichsel-(Würm)zeitliche Landschaft, 4. Die Ussing'sche Eisrandlinie, 5. Die Harder'sche Eisrandlinie, 6. Tunneltäler, 7. Das Odder-Elbo-Tal. 
mit Seen- oder Beckenablagerungen gefüllt. Im allgemeinen weisen sie heute ein Gefälle zum Zentrum des ehemaligen Eises hin auf. Die Tunneltäler sind durchschnittlich $1 / 2$ bis $3 \mathrm{~km}$, die ostjütischen Außenförden jedoch bis zu 5-10 km breit. Die Hauptrichtung der großen jütländischen Tunneltäler ist in Nordjütland NE-SW, im übrigen Jütland im Wesentlichen E-W.

Mit Ausnahme einiger - bezüglich ihrer Genese unsicherer - Täler ist die Hauptrichtung überall parallel zur regionalen Bewegungsrichtung des Inlandeises (und wahrscheinlich auch parallel zur Richtung des Eisabbaues). Wie es scheint, sind die Tunneltäler nördlich Djursland (Abb.1) schmaler als die Täler weiter südlich, wo sie, wie bereits vermerkt, hauptsächlich E-W gerichtet sind. Die Ursache hierzu ist wahrscheinlich im letzten Eisvorstoß bis zur HARDER'schen Eisrandlinie zu suchen, da eine Ubereinstimmung zwischen der Ausbreitung dieses Eises und der Verteilung der breiten Tunneltäler besteht.

Die glazialstratigraphischen Untersuchungen der letzten Jahre in Dänemark haben in Übereinstimmung mit Harder (1908) bewiesen, daß die Harder'sche Eisrandlinie (oder Ostjütländische Eisrandlinie) einem selbständigen Eisvorstoß zuzuordnen ist (BERTHELSEN 1973; SJÖRRING 1974, 1977). Zu diesen Untersuchungsergebnissen stehen allerdings die Auffassungen anderer Verfasser im Widerspruch (z. B. K. Milthers 1942; S. Hansen 1965; Nielsen 1967 und Marcussen 1977), die der Meinung sind, daß die HARDER'sche Linie nur eine Abschmelzlinie repräsentiert.

Aufgrund der erwähnten glazialstratigraphischen Untersuchungen konnten wir feststellen, daß die Tunneltäler östlich der HARDER'schen Eisrandlinie zumindest zum Teil älter sind als der der genannten Eisrandlage zugeschriebene letzte Eisvorstoß. Diese älteren Tunneltäler, die im W weit über die HARDER'sche Linie hinausreichen, wurden östlich dieser Linie vom erwähnten letzten Eisvorstoß überprägt. Auch Nordmann (1959) hat auf diese Zusammenhänge hingewiesen.

Von den klassischen Auffassungen über die Entstehung von Tunneltälern abgesehen, wurden in den letzten Jahren in Dänemark eine Reihe weiterer Gesichtspunkte diskutiert. Nordmann (1959) teilte mit, daß sich die subglazialen Schmelzwasserströme seitlich verschoben haben (vielleicht aufgrund der dämmenden Wirkung herabfallender Eisblöcke).

In einer Abhandlung über die Tunneltäler in Dänemark und Norddeutschland hat K. HANSEN (1971) frühere Auffassungen besprochen. Er macht darauf aufmerksam, daß die höchsten Punkte der Sanderkegel und die Enden der Tunneltäler sich nicht immer in übereinstimmender Lage zueinander befinden. Die Ergebnisse von Weiss (1958) und JASPERSEN (1953) zeigen, daß bei Berücksichtigung der Korngrößenverteilungen in den Sandern und der hydrologischen Verhältnisse im Eis ein genetischer Zusammenhang zwischen subglazialen Flüssen und Sandern nicht mehr gefolgert werden kann. Von hydrologischen Gesichtspunkten ausgehend, scheint man die klassische Tunneltalhypothese aufgeben zu müssen. Aufgrund seiner Studien schließt K. Hansen (1971), daß mehrere der Täler bereits saale-(riß-)zeitlich angelegt und später durch selektive Erosion übertieft wurden. Ahnliche Auffassungen bezüglich der selektiven Erosion äußerten auch WoLDSTEDT (1961) und GRIPP $(1964,1975)$.

Eine neue Hypothese über die Entstehung einiger Tunneltäler hat BERTHELSEN (1972) dargelegt. Aufgrund der Eisbelastung (Abb. 2) wird der Untergrund unter dem Eis herabgedrückt (Abb 2 B). Der dabei auftretende isostatische Druck in der Erdkruste unter dem Eis könnte einen Materialtransport aus dem Bereich unter dem Eis zum Eisrand erzwingen. Falls dies geschieht, müßte dies zur Bildung eines eisrandparallelen Wulstes führen (Abb. $2 \mathrm{C}$ ). Wenn dann der weitere Eisvorstoß rascher vonstatten geht als der beschriebene isostatisch bedingte Materialtransport, könnte das Eis auf den von ihm geschaffenen eisrandparallelen Wulst gelangen (Abb. 2 D). Schmelzwasser, die an dieser Phase 


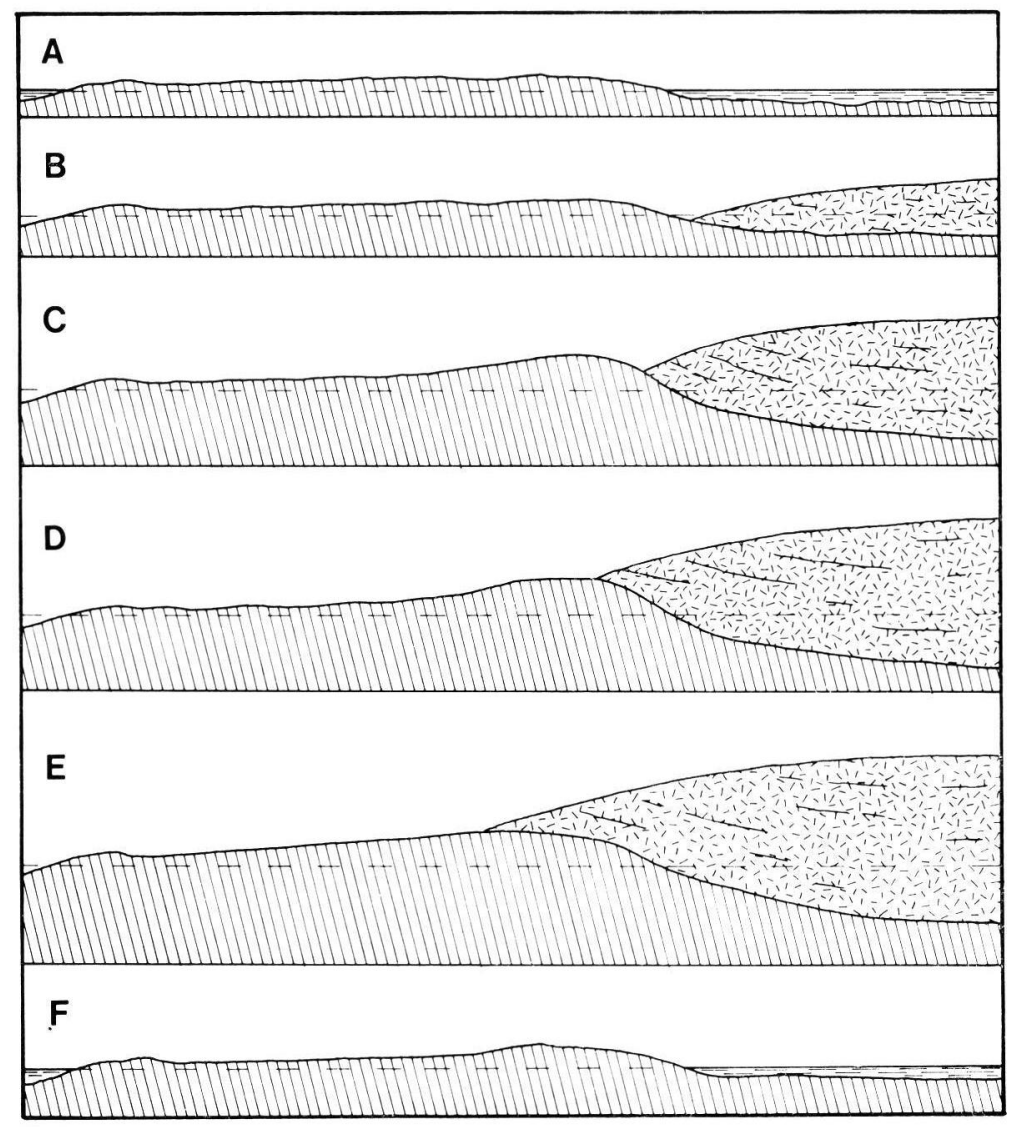

Abb. 2: Darstellung der hypothetischen Genese der Tunneltäler. Jütland im Querschnitt $(140 \mathrm{~km})$. Die Profile sind in fünfzigfacher Ưberhöhung gezeichnet. Erläuterung: siehe Text.

den Eisrand verlassen, fänden nun eine vom Eisrand weg gerichtete Oberflächenneigung vor und hätten in Richtung der Neigung subaerisch Täler erodieren können. Später waren die Täler bei Überschreitung durch das Eis durch selektive Erosion überprägt worden (Abb. 2 E).

Diese selektive Erosion könnte vielleicht mit Permafrost im Zusammenhang stehen, da dessen Tiefe unter Schmelzwasserflüssen geringer als in der Umgebung gewesen sein dürfte. Beim Vordringen des Eises könnte das unter der Permafrostzone einem entsprechend erhöhten Druck ausgesetzte Porenwasser unter Umständen im Bereich der Flußläufe nach oben dringen und dadurch die selektive Erosion begünstigen. Wenn diese Hypothese zutrifft, sollte es möglich sein, die alten Flußläufe unter den Sandern zu finden. Bisher hat nur Heller (1961) unter Sandern begrabene Täler in Westjütland beschrieben. Diese Täler könnten weichselzeitliche Bildungen oder aber auch älter sein.

Über das Alter der Tunneltäler liegen mehrere Informationen vor. $\Lambda$ NDERSEN (1973, 1974) und Schröder (1974) konnten nach Tiefbohrungen und geophysikalischen Untersuchungen in der Umgebung von Aarhus beweisen, daß unter einigen von ihnen über- 
prüften Tunneltälern und spätglazialen Entwässerungstälern ältere Täler vorkommen, die stellenweise Tiefen von mehr als $100 \mathrm{~m}$ unter dem Meeresspiegel aufweisen.

In mehreren dieser begrabenen Tunneltäler treten glaziale und interglaziale Sedimente auf. Dies bedeutet, daß die Täler nicht nur weichselzeitlich entstanden sind, sondern zum Teil nur weichselzeitlich überprägt wurden. ANDERSEN (1972) hat erwähnt, daß die tertiären Ablagerungen Jütlands im großen und ganzen nach SW einfallen. Er deutet an, daß alte Täler bereits im Tertiär gebildet wurden.

MARCussen (1977) vermutet, daß einige der Tunneltäler weichselzeitlichen Alters sind, da man an mehreren Stellen anerodierte Eemablagerungen in den Talhängen finden kann. Dazu ist zu bemerken, daß sich einige seiner Lokalitäten nicht in Tunneltälern, sondern in spätglazialen Entwässerungstälern befinden. MARCusSEN schließt jedoch nicht aus, daß einige der Tunneltäler älter sein könnten. Die alternative „Tunneltal“-hypothese (Niedertau-tälern) von MARcussen (1977) scheint übrigens der Hypothese von HormanN (1967), die von GRIPP (1974) besprochen wurde, zum Verwechseln ähnlich zu sein.

In Dänemark hat das Odder-Elbo-Tal (Abb. 1 u. 3) eine Sonderstellung als „Tunneltal" gewonnen. Dieses Tal weist eine ganz abweichende Richtung von den übrigen Tunneltälern im selben Raum auf. K. Milthers (1942) meinte, daß das Odder-Elbo-Tal längere Zeit mit Toteis gefüllt war, da es einen späteren kreuzenden Eisvorstoß überleben konnte.

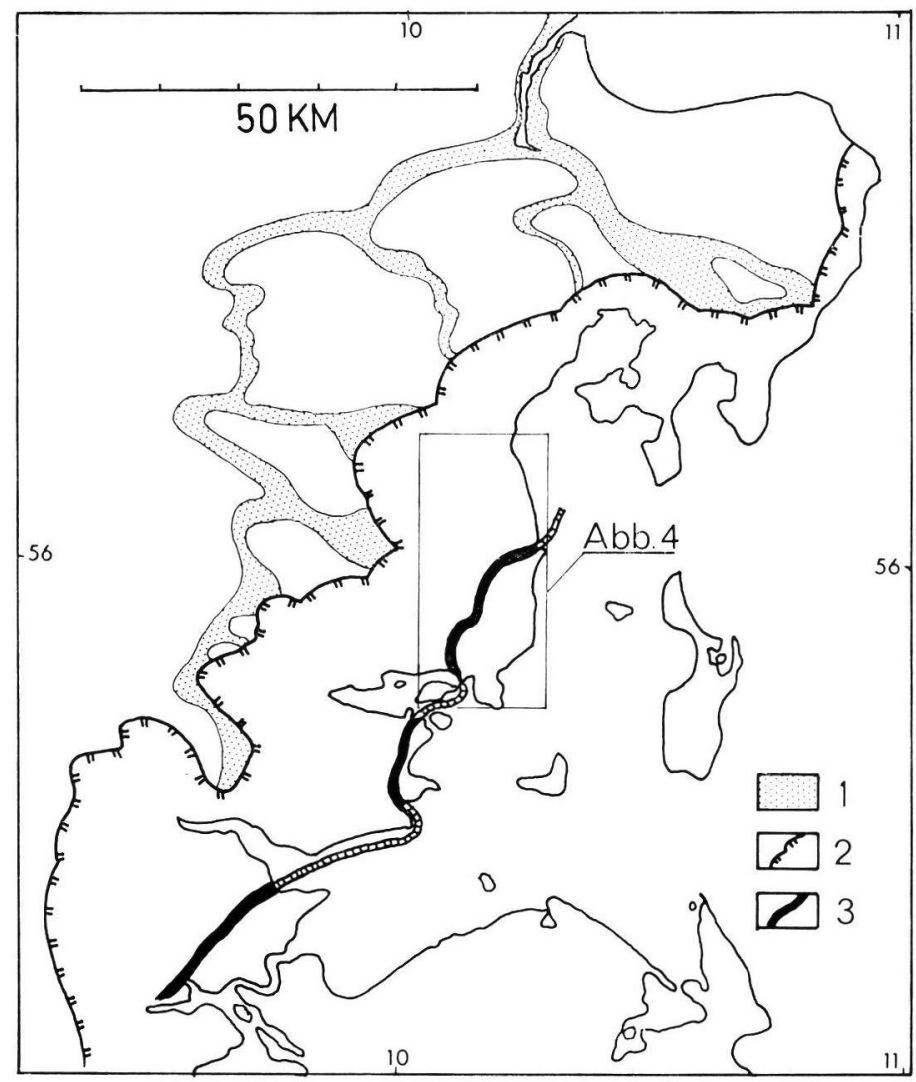

Abb. 3: Das Odder-Elbo-Talsystem (frei nach S. Hansen, Nielsen 1960 und Berthelsen 1972). Zeichenerklärung: 1. Sander und Entwässerungstäler des jungbaltischen Eisvorstoßes, 2. Die HARDER'sche Eisrandlinie, 3. Das Odder-Elbo-Tal. 


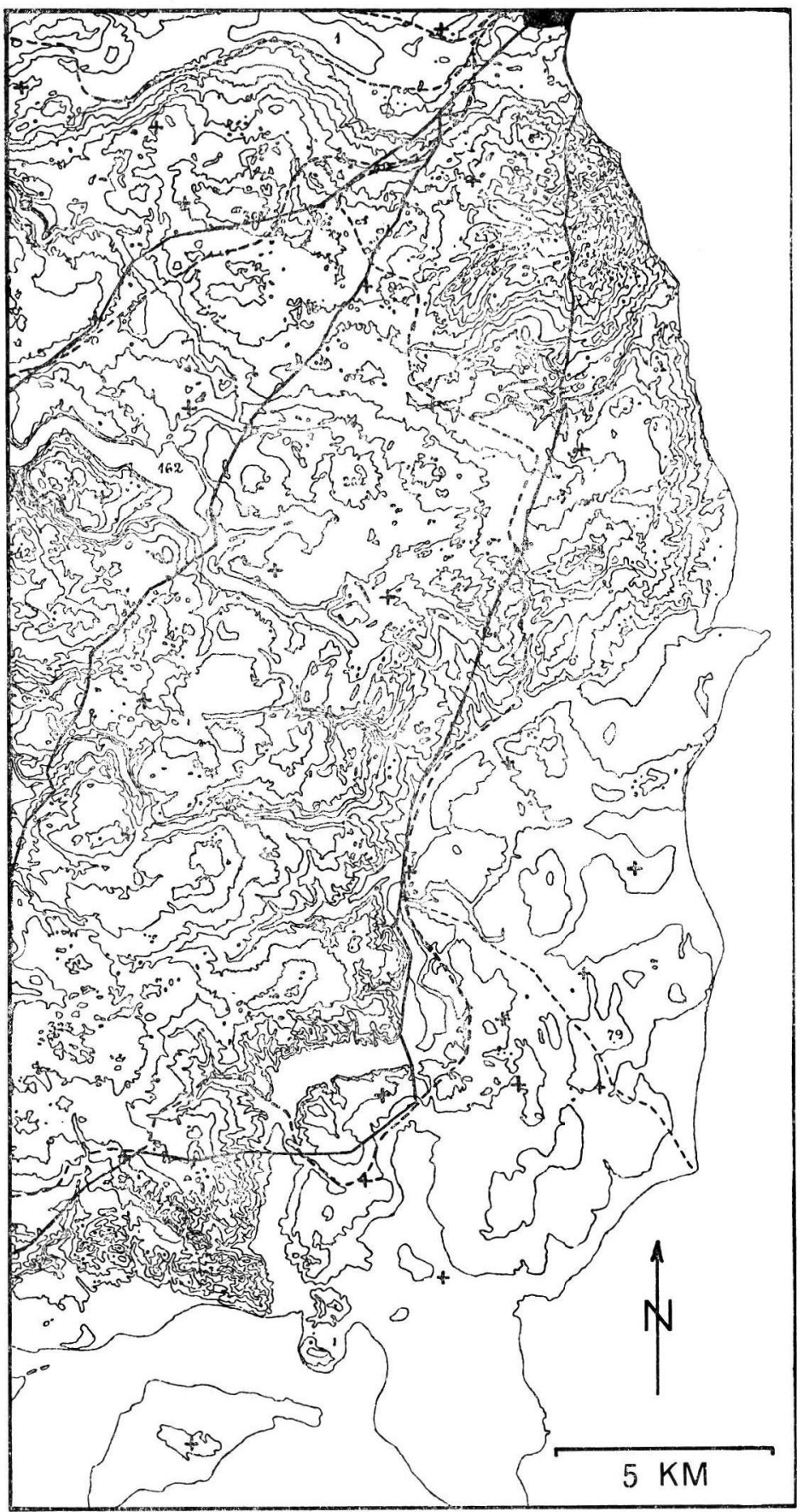

Abb. 4: Höhenliniekarte im Raum südlich von Aarhus (Ausschnitt nach Harder 1908). Lokalisierung s. Abb. 3. Höhenlinieabstand: 30 Fuss $=9,4$ Meter. Ausgezogene und gestrichelte Linien sind Hauptwege und Eisenbahnstrecken; Kreuze darstellen Kirchen. 

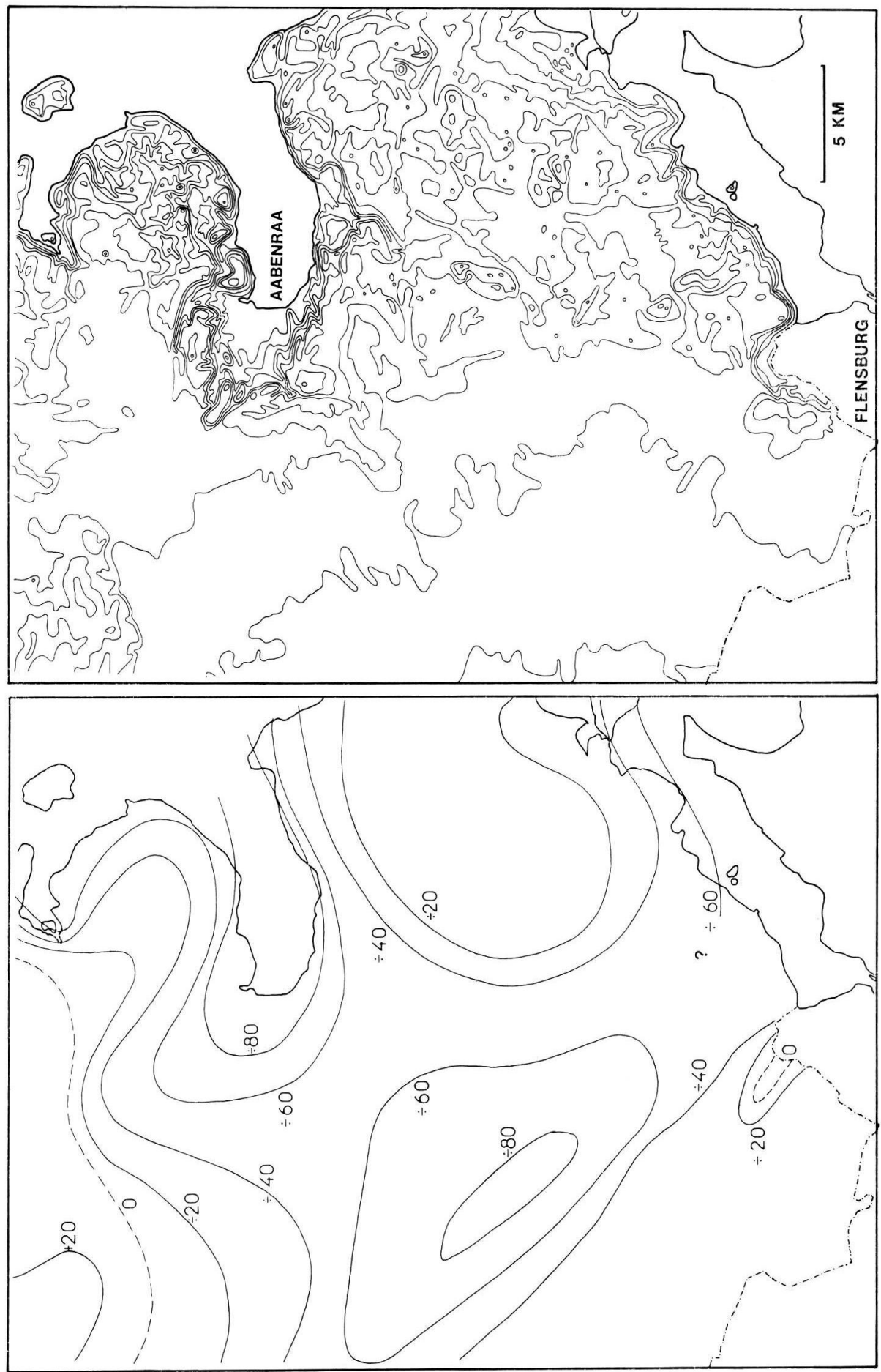

Abb. 5: Rechts: Höhenliniekarte in der Umgebung von Aabenraa (nach Jessen 1945), Höhenlinieabstand: 10 Meter. Links: Höhenliniekarte des präquartären Untergrundes (nach RASMUSSEN 1960) in demselben Gebiet. 
Berthelsen (1972) nimmt an, daß es sich um ein Urstromtal handeln könnte. Er folgert dies aus dem Umstand, daß es genau parallel mit dem Gudenaa-Talsystem verläuft, das als spätglaziales Entwässerungssystem der HARDER'schen Eisrandlage gedeutet wird. Auch die Morphologie weist darauf hin (Abb. 4), daß das Odder-Elbo-Tal eher ein extramarginales Tal ist als ein Tunneltal.

Die breiten Tunneltäler in Jütland, innerhalb der HARDER'schen Eisrandlage, sind ohne Zweifel von dem dieser Eisrandlage zugehörigen Eisvorstoß glazial übertieft worden (Abb. 5). Wahrscheinlich wurden die Tunneltäler außerhalb dieses Eisvorstoßes (Abb. 1 u. 5) ebenfalls durch selektive Erosion (in diesem Fall durch einen älteren Eisvorstoß bis zur Ussing'schen Eisrandlinie) überprägt. Sie sind aber ursprünglich durch aquatische Erosion proglazial (oder präglazial) angelegt worden und wahrscheinlich prä-weichselzeitlichen Ursprungs.

Die Tunneltäler auf Nordseeland haben ein anderes Aussehen (Abb.6). Sie können auch über begrabenen Tälern liegen, ähneln aber mehr einem Flußnetz im Toteis. Oft findet man in ihnen kleine Oser, von denen angenommen wird, daß sie in Spalten eines abschmelzenden Toteises gebildet wurden.

Für eine genaue Deutung dieser verschiedenen Täler benötigen wir neue Untersuchungen die Glazialstratigraphie und isostatische Bewegungen besonders berücksichtigen.

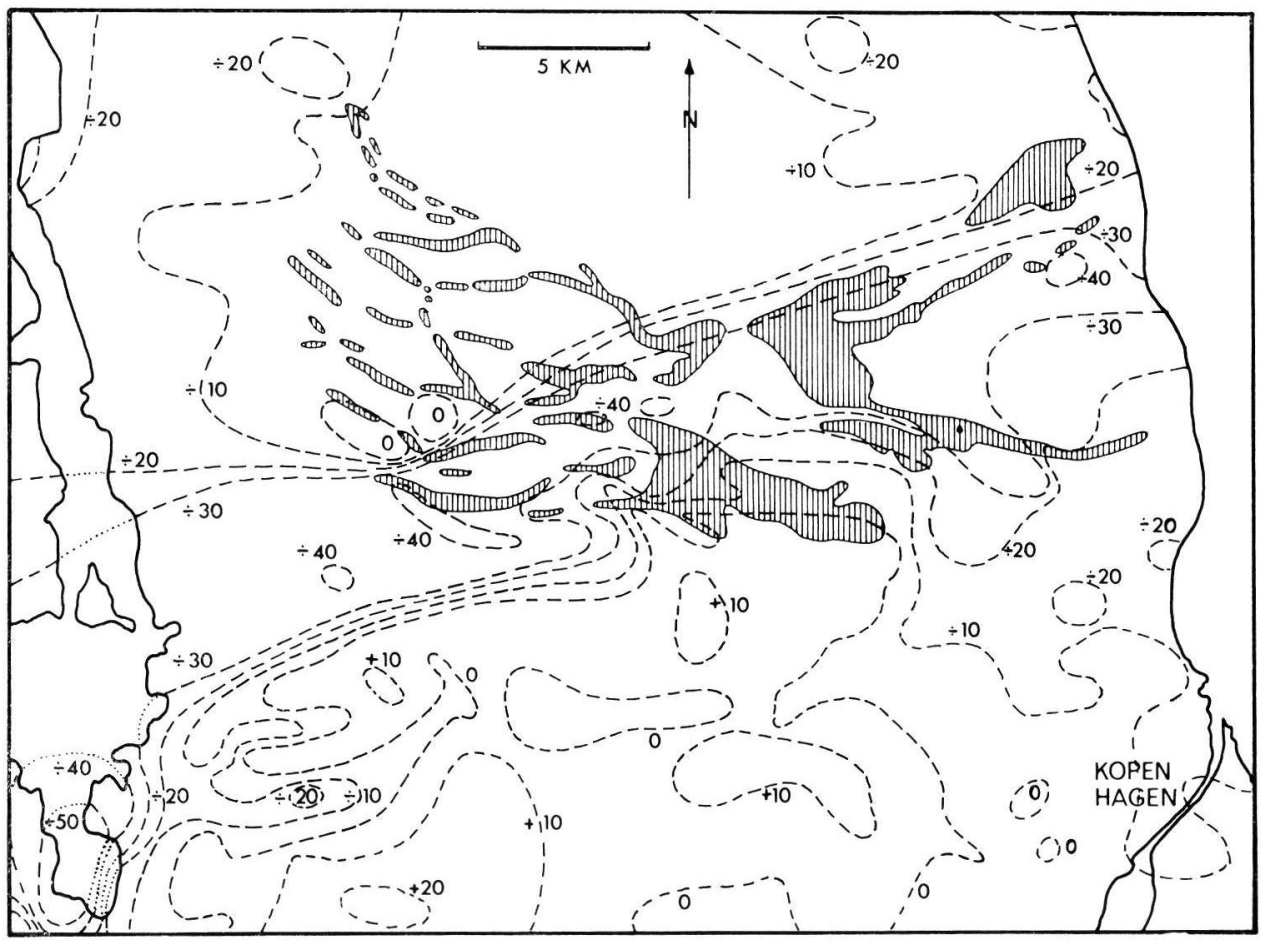

Abb. 6: Kartenausschnitt von Nordseeland (frei nach V. Milthers 1935). Gestrichelte Kurven sind Höhenlinien des präquartären Untergrundes (Abstand in Meter). Schraffierte Flächen darstellen Tunneltäler. 
Abschließend kann gesagt werden, daß wir in Zukunft den Ausdruck „Tunneltal“ nur mit größter Vorsicht gebrauchen sollten.

D a nk. Für kritische Diskussionen und für Hilfe bei der Übersetzung möchte ich Dr. Wa $1 \mathrm{t}$ er Vort is ch (Marburg) herzlich danken.

\section{Schriftenverzeichnis}

Andersen, H. Lykкe (1972): Viborgegnens tunneldale. - Museerne i Viborg Amt 2: 10-15; Viborg.

- (1973): En begravet dal i præ-Kvartæret ved Aarhus. Dansk geol. Foren., Aarsskrift for 1972: 111-118; København (Reitzel).

- (1974): Kortlægningen af undergrunden i omegnen syd og vest for Aarhus. - In: Laboratoriet for Geofysik (Hrsg.): Undersøgelser af Aarhusegnens undergrund, 57-101; Aarhus (Universitetet).

Berthelsen, A. (1972): Flod-, fjord- og tunneldale. - Dansk geol. Foren., Aarsskrift for 1971: 101-104; København.

- (1973): Weichselian Ice Advances and Drift Successions in Denmark. - Bull. geol. Inst. Univ. Uppsala, N.S., 5: 21-29; Stockholm.

Gripr, K. (1964): Erdgeschichte von Schleswig-Holstein. - 411 S.; Neumünster (Wachholz).

- (1974): Über die Schwierigkeiten, Vorgänge am Rand des quartären Inlandeises ohne entsprechende Kenntnis des heutigen Geschehens zu deuten. - Z. Geomorph. N.F., 18, 2: 224-229; Stuttgart.

- (1975): 100 Jahre Untersuchungen über das Geschehen am Rande des nordeuropäischen Inlandeises. - Eiszeitalter u. Gegenwart, 26: 31-73; Öhringen.

Hansen, K. (1971): Tunnel valleys in Denmark and Northern Germany. - Bull. geol. Soc. Denmark, 20: 295-306; Kopenhagen.

Hansen, S. (1965): The Quaternary of Denmark. - In: Rankama, K. (Hrsg.): The Quaternary, 1: 90 S.; New York, London (Interscience Publishers).

- \& Nielsen, A. V. (1960): Glacial Geology of Southern Denmark. - In: Sorgenfrei, T. (Hrsg.): International Geological Congress XXI Session in Norden; Guide to Excursions A 44 and C 39: 36 S.; Kopenhagen.

Harder, P. (1908): En østjysk israndslinie og dens indflydelse paa vandløbene. - Danm. geol. Unders. Rk II, 19: 259 S., und mit Atlas; Kopenhagen. [Summary in English: An ice-edge line in East Jutland and its influence on the water-courses].

Heller, E. (1961): Iagttagelser over tertiære og kvartære forhold i Tarm-Brande-Grindstedomraadet. - Meddr. dansk geol. Foren., 14:374-385; Kopenhagen. [Summary in English: Tertiary and Quaternary Observations in the Tarm-Brande-Grindsted Area].

Hormann, K. (1969): Gibt es Tunneltäler in Schleswig-Holstein? - Schr. Naturw. Ver. Schlesw.Holst., 39: 5-11; Kiel.

JASPERSEN, P. (1953): Sanderbildung durch subglaziäre, aufsteigende Schmelzwasserströme? Eiszeitalter u. Gegenwart, 3: 129-135; Ơhringen.

Jessen, A. (1945): Beskrivelse til Geologisk Kort over Danmark, Kortbladet Sønderborg. Danm. geol. Unders. Rh. I, 20: 91 S.; Kopenhagen. [Résumé en francais: Notice explicative de la feuille de Sönderborg].

Madsen, V. (1921): Terrainformerne på Skovbjerg Bakkeø. - Danm. geol. Unders. Rk. IV, 1 (12): 24 S.; Kopenhagen. [Résumé en francais: Les formes du terrain de la Colline Insulaire de Skovbjerg (en danois: Skovbjerg Bakkeø)].

Marcussen, I. (1977): Deglaciation landscapes formed during the wasting of the late Middle Weichselian ice sheet in Denmark. - Danm. geol. Unders. Rk. II, 110: 72 S.; Kopenhagen. [Dansk sammendrag: Deglaciations landskaber dannet under smeltningen af isskjoldet i sen mellem weichsel i Danmark].

Milthers, K. (1942): Ledeblokke og landskabsformer i Danmark. - Danm. geol. Unders. Rk. II, 69: 137 S.; Kopenhagen. [Summary in English: Indicator Boulders and Morphology of the Landscape in Denmark]. 
Milthers, V. (1935): Nordøstsjællands Geologi. - Danm. geol. Unders. Rk. V, 3: 192 S.; Kopenhagen.

Nielsen, A. V. (1967): Landskabets tilblivelse. - In: Nørrevang, A. \& Meyer, T. J.: Danmarks Natur, 1: 251-344; Kopenhagen.

Nordmann, V. (1959): Beskrivelse til Geologisk Kort over Danmark, Kortbladet Fredericia. Danm. geol. Unders. Rk. I, 22-A: 125 S.; Kopenhagen. [Summary in English: Explanation of sheet Fredericia].

Rasmussen, L. B. (1960): Molluscan Faunas and Biostratigraphy of the Marine Younger Miocene Formations in Denmark, Part I: Geology and Biostratigraphy. - Danm. geol. Unders. Rk. II, 88: 358 S.; Kopenhagen. [Dansk sammendrag: De danske marine yngre miocæne formationers molluskfaunaer og biostratigrafi, Del I: Geologi og biostratigrafi].

SchröDER, N. (1974): De geofysisk-geologiske undersøgelser af omraadet nord for Aarhus. In: Laboratoriet for Geofysik (Hrsg.): Undersøgelser af Aarhusegnens undergrund, 15-55; Aarhus (Universitetet).

SJöRRING, S. (1974): Über spätpleistozäne Glazialdynamik und -stratigraphie in Ost-Dänemark. - Eiszeitalter u. Gegenwart, 25: 208-209; Öhringen.

- (1977): The glacial stratigraphy of the island of Als, southern Denmark. - Z. Geomorph N. F., Suppl.-Bd., 27: 1-11; Stuttgart.

Ussing, N. V. (1903): Om Jyllands Hedesletter og Teorierne for deres Dannelse. - Overs. K. danske Vidensk. Selsk. Forh., 1903, 2: 99-165; Kopenhagen. [Résumé en francais].

- (1904): Danmarks Geologi i almenfatteligt Omrids. - Danm. geol. Unders. Rk. III, 2: 358 S.; Kopenhagen.

- (1907): Om floddale og randmoræner i Jylland. - Overs. K. danske Vidensk. Selsk. Forh., 1907, 4: 161-213; Kopenhagen. [Résumé en francais: Sur les Alluvions Glaciaires et les Moraines Terminales en Jutland].

Weiss, E. N. (1958): Bau und Entstehung der Sander vor der Grenze der Würmvereisung im Norden Schleswig-Holstein. - Meyniana, 7: 5-60; Kiel.

Woldstedt, P. (1913): Beiträge zur Morphologie von Nordschleswig. - Mitt. Geogr. Ges. u. d. Naturh. Mus. in Lübeck, 2 (26): 41-109; Lübeck.

Woldstedt, P. (1961): Das Eiszeitalter. I: 374 S.; Stuttgart (Enke).

\section{Nachtrag}

Während der Drucklegung erschien die Arbeit Kronborg, C., Bender, H. \& Larsen, G. (1978) Tektonik som en mulig medvirkende årsag til daldannelser i Midtjylland. - Danm. Geol. Unders., Årborg 1977: 63-76; Kopenhagen, in die die Entstehung von Tunneltälern in Zusammenhang mit Tektonik im Untergrund vorgeschlagen worden sind. 